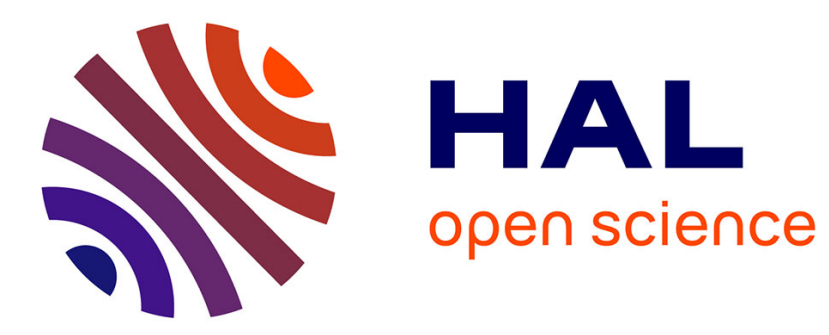

\title{
Chronique: Le congrès forestier régional des Alpes (16-19 juin 1964) - La tournée glaciollogique 1964 de la Société Hydrotechnique de France \\ - [.]revue Forestière Française, Rédaction
}

\section{- To cite this version:}

- [.]revue Forestière Française, Rédaction. Chronique: Le congrès forestier régional des Alpes (16-19 juin 1964) - La tournée glaciollogique 1964 de la Société Hydrotechnique de France. 1964, pp.871. $10.4267 / 2042 / 24603$. hal-03535475

\section{HAL Id: hal-03535475 https://hal.science/hal-03535475}

Submitted on 19 Jan 2022

HAL is a multi-disciplinary open access archive for the deposit and dissemination of scientific research documents, whether they are published or not. The documents may come from teaching and research institutions in France or abroad, or from public or private research centers.
L'archive ouverte pluridisciplinaire HAL, est destinée au dépôt et à la diffusion de documents scientifiques de niveau recherche, publiés ou non, émanant des établissements d'enseignement et de recherche français ou étrangers, des laboratoires publics ou privés. 


\section{CHRONIQUE}

\section{Le Congrès Forestier Régional des Alpes (16 au 19 juin 1964)}

Depuis sa création en 1947, le Fonds Forestier National a exercé son intervention en France à des titres divers, sur une étendue de terrains sans cesse croissante. Le cap du millionnième hectare bénéficiaire de cette intervention venant d'être franchi, M: le Ministre de l'Agriculture a décidé que 4 Congrès forestiers régionaux seraient tenus dans notre pays à cette occasion. Succédant aux Congrès qui eurent lieu au printemps dans les Landes de Gascogne et en Lorraine, précédant celui qui se tiendra cet automne dans le Sud du Massif Central, le Congrès forestier régional des Alpes se déroula du 16 au 19 juin 1964 en Dauphiné et en Savoie. Chacune de ces manifestations avait son thème particulier; le Congrès des Alpes était centré sur les questions d'équipement. Comme en montagne tout est solidaire: forêts, pâturages, rochers et torrents, neiges et glaciers, l'action du forestier s'est traditionnellement exercée dans ces régions, depuis plus d'un siècle, sur un théâtre fort élargi: en fait l'aménagement de l'espace alpin non agricole lui a été confié. C'est pourquoi les organisateurs du Congrès régional des Alpes, débordant quelque peu le cadre strict de l'équipement forestier, choisirent comme thème d'étude et de discussion: « Forêts, Alpages, Tourisme 》, avec l'idée non seulement d'examiner les actions forestières, passées en montagne, mais aussi de dégager si possible quelques lignes directrices pour l'avenir.

Le Congrès rassembla environ 150 participants: forestiers, propriétaires particuliers, exploitants, maires et conseillers municipaux de communes forestières, parlementaires et conseillers généraux, universitaires et professeurs, fonctionnaires appartenant à diverses administrations, etc... Il fut honoré par la présence des Directeurs Généraux des Eaux et Forêts des 6 pays du Marché Commun: Allemagne, Belgique, France, Italie, Luxembourg, Pays-Bas, certains d'entre eux accompagnés de leurs adjoints. Ils furent rejoints le dernier jour par plusieurs hauts fonctionnaires de la C.E.E. Monsieur le Ministre de l'Agriculture présida personnellement les manifestations de la dernière journée du Congrès à Annecy.

Le rassemblement des congressistes eut lieu à Grenoble. La journée du 16 juin fut consacrée à la visite de deux beaux massifs résineux de montagne des environs, mis en valeur par des réalisations routières qui en permirent l'exploitation rationnelle, auparavant difficile, voire impossible: la forêt communale de La Morte et le massif de Chamrousse - qui groupe 6 forêts communales ou domaniales distinctes en un ensemble qui dépasse 2300 hectares. A La Morte, le Maire, M. le Sénateur Mistral, accueillit les congressistes et présenta sa commune, typiquement montagnarde. A Chamrousse, outre le réseau routier, on visita le Centre montagnard de Jeunesse de Bachat-Bouloud, réalisation sociale d'avant-garde qui s'appuie sur la forêt. 
Ici comme là, on constata que l'ouverture des routes forestières avait fait doubler, voire tripler la valeur des bois sur pied, et que cette infrastructure routière avait été la base même du démarrage du tourisme d'altitude, aujourd'hui florissant à Chamrousse (station de séjour estival et de sports d'hiver), escompté pour demain à La Morte. Une agréable réception dans les jardins de la Préfecture clôtura cette première journée.

La matinée du 17 juin fut occupée par la visite de la route forestière d'Allevard (Isère) qui a elle aussi permis la mise en valeur d'un important massif résineux et le démarrage d'une petite station d'altitude: le Collet d'Allevard. $\mathrm{Au}$ terminus de la route, $\mathrm{M}$. PaQueT, député de l'Isère et Conseiller général, fit aux congressistes un exposé sur l'équipement touristique et sportif de la région d'Allevard et des Sept-Laux, promise à un grand avenir, et sur ses relations avec la forêt. Un rapide déjeuner eut lieu à Montmélian sous la présidence du Préfet de la Savoie, venu saluer spécialement le Congrès, qui ne faisait pourtant qu'effleurer son département sans y marquer d'autre arrêt.

L'après-midi du 17, on visita le massif du Semnoz, qui domine directement la ville d'Annecy et son lac. On gravit la nouvelle route de Leschaux au Crêt de Châtillon, aui traverse la forêt communale de Leschaux, ravagée par les chablis en 1962; cette route fut ouverte à point pour permettre de vendre à un prix raisonnable $\left(37 \mathrm{~F}\right.$ le $\left.\mathrm{m}^{3}\right) 8680 \mathrm{~m}^{3}$ de chablis qui, autrement, auraient pourri sur place en grande partie, les mieux placés seuls trouvant preneur à un prix dérisoire (autour de $8 \mathrm{~F} \mathrm{le} \mathrm{m}^{3}$ ). Au sommet du Semnoz, on eut un aperçu à la fois sur le complexe routier du massif, réalisé entre 1951 et 1963 . et sur la montagne pastorale qui couronne les forêts et occupe plus de 250 hectares. Cette exploitation pastorale. restée très vivante, a été grandement améliorée par la commune propriétaire avec l'appui du Service forestier: construction de chalets d'alpage modernes, adductions d'eau, lutte contre les mauvaises plantes, etc... Au cours de la descente sur Annecy, on marqua un court arrêt au Crêt du Maure, et $M$. le Conservateur Plagnat y fit un exposé fort instructif sur l'historique de la forêt communale d'Annecy (148 ha), d'origine entièrement artificielle (1861-1877). qui constitue actuellement un joyau aux portes du chef-lieu de la Haute-Savoie.

La tournée du 18 juin conduisit d'abord les congressistes à Samoëns, où l'on visita la montagne pastorale des Saix. Au terminus du télébenne, M. MALGRAND, Maire et Conseiller général, fit un exposé sur l'aménagement des alpages, centré dans deux directions différentes: modernisation de l'exploitation pastorale (chalet, étables, adduction d'eau, installation de purinage, lactoduc pour l'évacuation du lait vers la vallée), essor du tourisme (remontées mécaniques, chalet-refuge et chalets-hôtels, route en construction). L'aprèsmidi, on se rendit à Morzine pour examiner un complexe forestier - pastoral et touristique particulièrement typique, celui de Seraussaix - la Joue Verte - Avoriaz, véritable synthèse de ce qui avait été vu précédemment: mise en valeur d'une forêt d'accès très difficile, revivification d'une montagne pastorale excellente mais délaissée à cause de son isolement, démarrage du tourisme et des sports d'hiver dans un site magnifique. A Samoëns comme à Morzine, le Service forestier fut l'animateur de ces transformations, auxquelles un appui financier approprié fut accordé dans certains cas.

Durant ces trois journées de visites sur le terrain, les services forestiers locaux furent constamment sur la brèche: à chaque arrêt, des exposés techniques très étoffés, illustrés de nombreux plans et croquis, furent présentés aux congressistes par les ingénieurs responsables.

Le 19 juin fut consacré aux séances d'études à Annecy, dans la grande salle du Casino, parfaitement sonorisée. Sous la présidence de M. l'Ingénieur Général des Eaux et Forêts WidmanN, les rapporteurs particuliers présentèrent des exposés étoffés et exhaustifs: H. Bonnet-Masimbert sur l'équipement routier des forêts de montagne, L. ANCHIERRI sur les travaux d'amélioration pastorale et leur orientation actuelle, M. Bouverot sur la protection contre les avalanches, H. Huchon sur « Tourisme et forêt en montagne ». Des discussions suivirent chaque présentation de rapport, à la lu- 
mière des réalisations visitées les jours précédents. Le rapport général d'ensemble fut ensuite exposé par P. Fourchy, en essayant de tirer des conclusions valables, dont l'Administration puisse s'inspirer pour bâtir une politique d'avenir applicable en montagne.

Nous reviendrons sur ces questions dans des articles techniques spéciaux.

Un banquet, présidé par M. le Ministre de l'Agriculture entouré de nombreuses personnalités locales, clôtura le Congrès, qui fut favorisé de bout en bout par un temps magnifique... sauf pendant la séance d'étude en salle du dernier jour, ce qui était sans importance, l'attention se portant alors davantage sur les exposés des orateurs, semble-t-il, que sur l'état d'un ciel boudeur.

P. F.

\section{La tournée glaciologique 1964 de la Société Hydrotechnique de France}

La tournée glaciologique de la Société Hydrotechnique de France s'est déroulée cette année dans le Mercantour, du 21 au 25 septembre 1964, sous la direction de M. l'Ingénieur Général des Eaux et Forêts Messines. Une quarantaine de personnes y participaient, notamment un nombre appréciable d'ingénieurs des Eaux et Forêts et d'ingénieurs à l'Electricité de France, mais aussi des représentants de l'Université, de l'Institut géographique national, des Ponts et Chaussées, etc... On notait la présence de plusieurs étrangers, dont le Professeur VanNi, Secrétaire du Comité glaciologique italien, et trois ingénieurs forestiers espagnols.

Une journée fut consacrée à l'étude des phénomènes torrentiels et de l'économie montagnarde des vallées de Vars et d'Escreins (Hautes-Alpes). Après avoir visité la célèbre dérivation de Panacelle, sur le Chagnon, on se rendit à Escreins, d'où l'on passà dans la vallée de Vars en franchissant le Col de la Coulette et en parcouranit les pâturages communaux, puis en * descendant le cours du Chagnon, dont de nombreux ouvrages de correction régularisent le cours. M. le Conservateur des Eaux et Forêts Batias et MM. les ingénieurs Rogie et BASTIDE donnèrent de nombreuses explications techniques en cours de route. Le soir, M. Benard, député des Hautes-Alpes et maire de Vars, fit aux congressistes un exposé fort original sur l'équipement et le développement de sa commune, appelée à un grand avenir touristique.

Le mardi 22 fut occupé par la visite de l'un des rares glaciers existant dans le département des Basses-Alpes, celui du Marinet, plaqué sur la face Nord du Brec de Chambeyron, glacier dont l'étendue réelle reste contestée, à cause des nombreux éboulis qui le recouvrent dans sa partie inférieure. M. l'Ingénieur des Eaux et Forêts Bucher fit un exposé fort intéressant sur la question.

Le 23 , on gagna la vallée de la Vésubie par le Col de Restefond et l'on monta s'installer au refuge de Nice (alt.: 2232 mètres). C'est de ce point de départ qu'on partit pour visiter le lendemain les glaciers de la Malédia et du Clapier, sur le versant italien du Mercantour. M. l'Ingénieur Principal Bosio était l'organisateur de cette dernière partie de la tournée.

Le dernier jour fut consacré aux équipements hydroélectriques de la vallée de la Vésubie (chutes de la Gordolasque, de la Vésubie et du Boréon).

Favorisée par un temps magnifique et par un éclairage d'automne qui embellissait toutes choses, cette tournée fut une réussite. Indépendamment des précieux renseignements techniques qu'ils y puisèrent, les participants appartenant à des professions et à des nationalités variées - purent procéder à de fructueux échanges de vues sur les problèmes de la montagne qui les intéressent tous sous des aspects divers.

P. F. 